\title{
NEW RESEARCH IN SOLAR CELLS Urbach Tails and Open Circuit Voltage
}

MICHELLE SOLOMON AND ALISON JOHNSON

THE DEVELOPMENT OF VARIOUS EMERGING PHOTOVOLTAIC TECHNOLOGIES, AS CLASSIFIED BY THE NATIONAL RENEWABLE ENERGY LABORATORY, HAS EXPERIENCED VERY DIFFERENT RATES OF PROGRESS OVER THE LAST FIVE TO TEN YEARS. IN PARTICULAR, TWO THIN-FILM TECHNOLOGIES, QUANTUM DOT SOLAR CELLS AND PEROVSKITE-BASED SOLAR CELLS, HAVE ACHIEVED DRASTICALLY DIFFERENT VALUES FOR POWER CONVERSION EFFICIENCY AS QUANTUM DOTS HAVE FAILED TO KEEP UP WITH PEROVSKITES. CLEARLY, THE MATERIALS ARE VERY DIFFERENT, BUT IN ORDER TO QUANTIFY THE DISCREPANCY IN EFFICIENCY, WE FOCUS ON MEASURING THE TAIL OF THE ABSORPTION PAST THE NOMINAL BAND EDGE AND THEREFORE THE URBACH ENERGY OF THE TWO MATERIALS. WE DISCUSS A THEORETICAL RELATIONSHIP BETWEEN URBACH ENERGY AND OPEN CIRCUIT VOLTAGE BASED ON THE ORIGINAL CONSIDERATIONS BY SHOCKLEY AND QUEISSER ON THE EFFICIENCY LIMITS OF SOLAR CELLS. 
One of the main concerns of photovoltaic technology for the consumer is the cost, specifically the fabrication cost per unit of energy or power produced. One way to minimize the cost is to decrease the amount of material by using thin film solar cells. Thin films also have potential for nonconventional realizations of solar cells that require only simple mounting apparatuses, even as simple as a sticker. However, a key issue that arises with most thin films is a low power conversion efficiency compared to thick, crystalline cells. Both the path length of the light within the absorptive layer of the cell and the path length of electrons excited by the absorbed light decrease in a thin film, which creates a tension of optimization. The former feature makes it difficult for a thin film cell to absorb light, while the latter makes it easy for the electrons to get out as electric current. Thus, if the layer is made too thin, little light is collected, but if it is made too thick, the conversion efficiency again decreases.

This issue has stalled many thin film technologies, but emerging research is taking different approaches to attempt a solution. Two areas in particular, quantum dot solar cells and perovskite-based solar cells, have experienced similar popularity, but very different rates of progress toward increased power conversion efficiency.

Quantum dot photovoltaics first gained popularity in the early 2000 s, when it was predicted that quantum confinement should lead to a quantized density of states in the zero-dimensional nanoparticles. Such a property, in contrast to the quasi-continuous nature of energy levels in a bulk semiconductor, should then allow the electron relaxation time to increase, potentially allowing for either multi-exciton generation or extraction of hot electrons. This, in addition to the tunability of absorption into the near infrared region should allow for an unprecedented increase in efficiency of photovoltaics, up to $66 \% .{ }^{{ }^{1}}$ However, experiment has not nearly reflected this theoretical increase, as quantum dot solar cells have only reached a maximum efficiency of $8.55 \%$. $^{2}$

Lead-halide perovskites, in contrast, have seen rapid progress, up to the current confirmed record of $17.9 \%{ }^{3}$ Perovskites have a highly ordered crystal lattice that allows for good optical and electrical properties, but they are not unique in this characteristic. Crystalline silicon, for example, is similarly ordered. The interest in perovskites, then, stems from their potential as a particularly thin, and therefore cheaper, crystalline material. Perovskites have been synthesized with a diffusion length of up to I micron, which is an order of magnitude higher than the absorption depth, which can be as small as Ioo nm. Crystalline silicon, with its similar amount of order exhibits a longer diffusion length, but its optical properties are not as good, and its absorption depth nears Ioo microns in the Vis-NIR range that is of interest for photovoltaic applications. ${ }^{4}$

Here we will address a fundamental optical property, the Urbach energy, of the two materials (lead-halide perovskites and lead sulfide quantum dots) that can provide a more quantitative explanation for the discrepancy in the efficiencies seen in the two materials.

\section{THEORY}

The Urbach energy is connected to the theoretical limit of the efficiency of a single p-n junction solar cell, but goes past the treatment given by Shockley and Queisser in their famous detailed balance limit. This limit, initially derived for material with a band gap of I.I eV, gives a value given for efficiency, $\eta$, that depends on four terms:

$$
\eta\left(\chi_{g}, \chi_{c}, f, t_{s}\right)=u_{g}\left(\chi_{g}\right) v\left(\chi_{g}, \chi_{c}, f\right) m\left(\chi_{g}, \chi_{c}, f\right) t_{s}
$$

The first term, $u_{o}$, is defined as the ultimate efficiency, and represents the efficiency the cell would have if every photon with energy above the band gap of the material in question were to excite an electron that would then provide the exact energy of that photon to the cell. The third is an impedance matching factor that accounts for the balance between the highest possible values for voltage and current, which cannot occur simultaneously. The fourth is the probability that a photon with energy greater than $\mathrm{E}_{\mathrm{g}}$ will excite an electron and produce and electron hole pair; the value's upper limit is I.

The second term, $\mathrm{v}$, is the ratio of the operational output voltage to that of the band gap, and it is of particular interest here. It accounts for recombination processes in the cell, making considerations for both radiative and nonradiative recombination.

To find the maximum value for the open circuit voltage that a solar cell can attain, and therefore the ratio of that open circuit voltage to that of the band gap, Shockley and Queisser first consider the steady-state condition that will occur when the cell is surrounded by a blackbody of temperature $T_{c}$. In this state, the rate of radiative recombination is given by a value $\mathrm{F}_{\mathrm{co}}$. This is determined by the num- 
ber of photons incident upon the cell with energy greater than $\mathrm{E}_{\mathrm{g}}$, which is derived from the blackbody spectrum at $\mathrm{T}_{c}$. When solar radiation hits the cell, the original state is disrupted and the new steady-state is given by

$$
0=F_{s}-F_{c}(V)+R(0)+R(V)-\frac{I}{q}
$$

where $F_{S}$ is the rate of electron-hole pairs generated due to solar radiation (and is determined by the blackbody curve of a body at $6000 \mathrm{~K}), F_{c}(V)$ is the new rate of radiative recombination, $R(0)$ is the rate of radiative recombination, $R(V)$ is nonradiative generation, and $I$ is the external current. In the ideal situation, nonradiative processes will obey the ideal rectifier equation, and

$$
R(V)=R(0) \exp \left(\frac{V}{V_{c}}\right)
$$

The $F_{c}(V)$ term is determined by the new concentration of electrons and holes after the Fermi-level split into quasilevels due to the incident radiation, and is therefore defined as

$$
F_{c 0}=F_{c 0} \exp \left(\frac{V}{V_{c}}\right)
$$

Equation (I) can also be written as

$$
0=F_{s}-F_{c 0}+\left[F_{c 0}-F_{c}(V)+R(0)+R(V)\right]-\frac{I}{q}
$$

where the term in the brackets represents the net rate of generation of electron-hole pairs when a cell is surrounded by a blackbody at temperature $T$, since $F_{s}-F_{c o}$ will be zero at that point. Using this term, the fraction of recombination that will be radiative can be written as

$$
f_{c}=\frac{F_{c 0}-F_{c}(V)}{F_{c 0}-F_{c}(V)+R(0)-R(V)}
$$

The steady-state can now be expressed as

$$
0=q\left(F_{s}-F_{c 0}\right)+\frac{q\left(F_{c 0}-F_{c}(V)\right)}{f_{c}}-I
$$

Setting $I$ equal to zero in order to find the maximum value for $V$, and using the definition in (3)

$$
F_{s}-F_{c 0}=\frac{F_{c 0}}{f_{c}}\left(e^{\frac{V}{V_{c}}}-1\right)
$$

Solving for V,

$$
V=V_{c} \ln \left(\frac{f_{c} F_{s}}{F_{c 0}}-f_{c}+1\right)
$$

Since $F_{s}$, based on the blackbody curve at $6000 \mathrm{~K}$, is much greater than all other terms, as long as $f$, which is assumed to be I in the final calculation, is not too small. ${ }^{5}$

The absorption coefficient for solar energy striking the surface of the cell is also assumed to be I. In other words, the assumption is made that photons with energy higher than $E_{\mathrm{g}}$ are absorbed and excite an electron with a probability of $\mathrm{I}$, while photons with energy below $E_{\sigma}$ are absorbed and excite an electron with a probability of o. However, this assumption does not hold for the low values of $f_{c}$ that the detailed balance limit does not consider. In such a case there will be an exponential tail present in the absorption spectrum of the material being studied. Highly ordered crystalline materials exhibit very sharp absorption edges, but for both amorphous materials and films of quantum dots the disorder leads to a broad absorption tail below $E_{g}$. The breadth of this absorption tail is often well described by a value $E_{u}$, the Urbach energy, and an exponential such that

$$
\alpha(E)=\alpha_{0} \exp \left(\frac{E-E_{o}}{E_{u}}\right)
$$

The open circuit voltage of the cell, when the absorption tail is considered, can now be represented $\mathrm{as}^{6}$

$$
V_{o c}=\frac{k T}{e}\left(\ln \left(\frac{\int_{0}^{\infty} \alpha(E) \Phi_{s}(E, T) \mathrm{d} E}{\int_{0}^{\infty} \alpha(E) \Phi_{T}(E, T) \mathrm{d} E}\right)+1\right)
$$

Here, $\alpha(E)$ is defined as the exponential absorption edge below $E_{g}$ and I above $E_{g}$. When $V_{o c}$ is calculated for different values of $T$ and $E_{u}$, this inverse relationship becomes clear.

As is evident from the calculations, $E_{u}$, when greater than $k_{B} T$ at different values of $T$, leads to a significant decrease in $V_{O C}$ compared to the given value of $E_{g}$, which was taken to be I. $3 \mathrm{eV}$ for the purpose of the calculations. Also noteworthy is the trend toward zero of the difference $E_{g}-V_{O C}$ as both $T$ and $E_{u}$ go toward zero as well, which is in agreement with the predictions of Shockley and Queisser.

Literature data, gathered by De Wolf et al., show a nearly linear relationship between Urbach energy and losses in $V_{O C}$. These are shown in Figure 2 along with the calculated value from Eq.Io. Except for one point, the calculated $V_{O C}$ is larger than the experiment which is fine since the calculation does not introduced additional efficiency losses. The same positive relationship can be seen between $E_{u}$ and losses in $V_{O C}$ in both calculation and experiment.? 
This relationship indicates that the value for the Urbach energy should give a prediction as to the upper limit that the open circuit voltage of solar cell can attain based on its material characteristics, taking into consideration more than simply the band gap of those materials.

\section{METHODS}

To confirm the value of this prediction, we compare Eu for quantum dots and perovskites, specifically lead sulfide dots with absorption peak near $950 \mathrm{~nm}$ and lead-halide perovskites with absorption edge near $800 \mathrm{~nm}$, in order to better understand the power conversion efficiencies that have already been attained with such materials.

The synthesis used for the lead sulfide quantum dots is based on a modified version of the Hines and Scholes method. ${ }^{8,9}$ Briefly, 0.09 g PbO, $0.27 \mathrm{~mL}$ oleic acid, and 3.8 $\mathrm{mL}$ octadecene (ODE) were mixed under a Schlenck line in a three-neck flask. The mixture was degassed at 95I0 $5^{\circ} \mathrm{C}$ for Io minutes and then heated to $150^{\circ} \mathrm{C}$ under $\mathrm{Ar}$ gon for another 50 minutes. $42 \mu \mathrm{L}$ of bis(trimethylsilyl) sulfide (TMS) was dissolved in $3 \mathrm{~mL}$ ODE in a glovebox and then injected into the heated solution. The mixture turned to a dark brown within a second of injection. The temperature dropped to around $1 \mathrm{I} 5^{\circ} \mathrm{C}$ at which it was allowed to react for 20-30 seconds. The flask was cooled back to room temperature using air flow. The quantum dots were precipitated with ethanol and/or acetone, and dissolved in a 9:I hexane/octane solution.

The lead sulfide substrates were prepared by cross-linking with ethanedithiol (EDT). A glass slide was cleaned using acetone and ethanol. The glass was then covered with 3-(mercaptopropyl) trimethoxysilane (MPTS) to bind the quantum dots to the glass. The methoxy groups bind to the glass and the thiol groups are available to bind to the $\mathrm{Pb}$. The cleaned $\mathrm{PbS}$ QDs were dropped onto the glass slide, which formed a thin film on the surface. The film was covered with EDT in ethanol for a few seconds before washing with ethanol. The films were dried and the process was repeated until a dark, smooth layer was formed. These films were measured with the Cary UV-Vis or PDS before any degradation from air could take place.

The method for the synthesis of lead iodide perovskites was based on that used by Im, et al. ${ }^{\text {IO }}$ The methylammonium iodide precursor was prepared by reacting methylamine (2.0 $\mathrm{M}$ in methanol) and hydriodic acid $(57 \%$ in water) for 4 hours at $\circ^{\circ} \mathrm{C}$. The methylamine solution in methanol was prepared by bubbling methylamine gas through $223 \mathrm{~mL}$ of methanol while swirling the flask. This resulted in a I-2 $\mathrm{M}$ solution. The HI was added slowly drop-wise to the methylamine which had been cooled to $\circ^{\circ} \mathrm{C}$. The solution changed color from clear to yellow upon addition of $\mathrm{HI}$, and the solution was a reddish hue by the end of the 4 hour reaction. The precipitate was collected using a rotary evaporator overnight at $80^{\circ} \mathrm{C}$. The precipitate was redissolved in $3.5 \mathrm{~mL}$ ethanol at $70^{\circ} \mathrm{C}$ and recrystallized to remove impurities. The crystals were washed with diethyl ether and filtrated. Because the crystals still had a yellow tinge, they were recrystallized until a white powder was obtained. The powder was dried overnight in a vacuum oven at $100^{\circ} \mathrm{C}$. In a glovebox, approximately 0.5 $\mathrm{g}$ of lead iodide and I.3 $\mathrm{g}$ of methylammonium iodide were dissolved into $2.3 \mathrm{~mL} \gamma$-buteralactone, heated to $80^{\circ} \mathrm{C}$, and reacted for 2 hours. The perovskites were then spin coated onto glass coated with polyethleneamine and rinsed with water at $6000 \mathrm{RPM}$, and baked at $100^{\circ} \mathrm{C}$ for $\mathrm{I} 5$ minutes. The perovskites were never exposed to air throughout the synthesis and measurements.

To measure the absorption spectrum of the materials, and therefore the Urbach energy, photothermal deflection (mirage) spectroscopy (PDS) was used. The measurements were done using a Ioo W tungsten lamp as a white light source, a monochromator based on a 1200 groove $/ \mathrm{mm}$ diffraction grating blazed at $1000 \mathrm{~nm}$ and I $\mathrm{mm}$ slits, and a $635 \mathrm{~nm}$ red laser. The resolution of the monochromator was calculated to be approximately $0.7 \mathrm{mV}$ (where the slit width was taken to be I $\mathrm{mm}$ and the focal length was taken to be Io $\mathrm{cm}$ ), which is well within the resolution needed to measure the steepest absorption tail expected, around Io$20 \mathrm{mV}$. The white light is modulated at a frequency of 20 $\mathrm{Hz}$, and after passing through the monochromator, is focused to a $5 \mathrm{~mm}$ by $0.5 \mathrm{~mm}$ area on a $5 \mathrm{~mm}$ wide sample, which is immersed in filtered hexane and sealed in an airtight quartz cuvette. The red laser skims the surface of the sample perpendicular to the diffracted monochromatic light. Therefore, when the sample absorbs a certain wavelength of light, it heats the hexane, changing the index of refraction and bending the red laser beam. The change in position of the red laser beam is the direct measurement, from which the absorption spectrum is obtained after normalization with the spectrum of the lamp measured using an opaque graphite sample. Filters are used to block the second order diffraction and extend the measurement into the near infrared region. The absorption spectrum can be plotted on a logarithmic scale, with the steepest slope corresponding to the minimum value of $E_{u}$ 


\section{RESULTS AND DISCUSSION}

The absorption spectrum was measured for $\mathrm{PbS}$ quantum dots using PDS, and is shown in Figure 3. There are two distinct linear areas when plotted on a logarithmic scale, corresponding to two different tails. The minimum slope is $98 \mathrm{meV}$, measured through an order of magnitude, which is therefore the minimum Urbach Energy measured for these dots.

This value for the Urbach energy, at room temperature, can be calculated to correspond to losses in $V_{O C}$ from $E_{g}$ in the range of $0.8 \mathrm{~V}-\mathrm{I} .0 \mathrm{~V}$ at room temperature, $270 \mathrm{~K}$ (see Figure I). This is further supported by experimental values; the maximum reported value of $V_{O C}$ for a $\mathrm{PbS}$ quantum dot solar cell, to our knowledge, is $0.692 \pm$ $0.007 \mathrm{~V}$, attained from dots of $2.9 \mathrm{~nm}$ with a band gap of I. $4 \mathrm{eV}$ - a loss of $0.7 \mathrm{~V}$. A range of dots of different sizes and band gaps were fabricated and measured by Yoon, et al., as seen in Figure $4 .{ }^{\text {II }}$

A linear fit to these points gives the relation

$$
\mathrm{V}_{\text {OC }}=0.519\left(E_{\mathrm{g}}\right)-0.022 \mathrm{I}
$$

which indicates that the maximum open circuit voltage achieved from a photovoltaic device fabricated from $\mathrm{PbS}$ quantum dots has only slightly exceeded approximately $50 \%$ of the measured value of $E_{g}$, which, in order to absorb in the desired region of the near infrared, is generally near I.3 V, as is the band gap in the dots that we fabricated as well.

This relationship holds true not only for high voltage cells, but also for high efficiency cells, which is logical since $\eta$ depends on $V_{o c}$. The highest reported efficiency for a $\mathrm{PbS}$ solar cell to date is $8.55 \%$, which was achieved with dots with $E_{\sigma}$ I.3 V. The maximum open circuit voltage attained for these cells was $0.6 \mathrm{~V}$, using dots capped by I,2 and I,3-benzenedithiol ligands. Again, we see a loss of about $0.7 \mathrm{~V}$ from the band gap voltage for a lead sulfide quantum dot solar cell. ${ }^{2}$

The width of the Urbach tail is indicative of the level of disorder in a material, which for quantum dots, with a high surface area to volume ratio, is fundamentally connected to the nature of the surface of the dots. Imperfections in stoichiometry and passivation at the surface, as well as the type of ligands that cap the dots are often the most significant sources of disorder. It is therefore pos- sible to increase the sharpness of the tail by optimizing the types of ligands used. For example, different ligands can either passivate the surface more or less efficiently, affecting the amount of trap states on the surface. Furthermore, Urbach energy generally increases with increasing ligand length, possibly due to decreased coupling between dots. ${ }^{12}$ The effect of different ligands on the Urbach energy in CdSe dots has been explored using PDS, and was found to be significant, with CdSe dots caped by HDA exhibiting a minimum $E_{u}$ of $25 \mathrm{meV}$, while the same dots capped with $\mathrm{S}^{2 \cdot}$ had an increased minimum Urbach energy of $80 \mathrm{meV}^{\mathrm{I3}}$ Clearly, then, there is some potential in this area for improvement in the absorption tail of quantum dots, but attempts at optimizing ligand use has not lead to the increases in efficiency that were originally predicted, indicating that the Urbach energy for PbS QDs is still too high to attain the necessary values for $V_{O C}$.

Perovskites, with their crystalline structure, are inherently more ordered than quantum dots, leading to a much lower value for the Urbach energy, and therefore higher values for the open circuit voltage. For example, the highest value for open circuit voltage attained up to this point is I.4O V, which was achieved using a wide band gap bromine based lead perovskite with a band gap energy of $2.30 \mathrm{~V}$. To make a more direct comparison to measurements made here, the highest value achieved for $\mathrm{CH}_{3} \mathrm{NH}_{3} \mathrm{PbI}_{3}$, is $\mathrm{I} .05 \mathrm{~V}$, from a band gap of I.57 V. ${ }^{\mathrm{I}}$ The most efficient perovskite cells have been based on mixed-halide perovskites, of the form $\mathrm{CH}_{3} \mathrm{NH}_{3} \mathrm{PbCl}_{\mathrm{x}} \mathrm{I}_{3-\mathrm{x}}$, which have the same band gap as pure iodide perovskites, but a higher reported value for $V_{O C}$, at I.I3 V. 15

The absorption spectrum measured for $\mathrm{CH}_{3} \mathrm{NH}_{3} \mathrm{PbI}$ using PDS is shown in Figure 5. The exponential curve is fitted to a region that stretches through two orders of magnitude, giving a value of $45 \mathrm{meV}$ for $E_{u}$. This is somewhat higher than the lowest reported value of $15 \mathrm{meV}$, but it is substantially lower than the measured value of $98 \mathrm{meV}$ for the PbS QDs. When compared with the highest reported value for $V_{O C}$ above, it agrees well with the calculation, which predicts that an Urbach energy of $45 \mathrm{meV}$ should give a loss of just over $0.5 \mathrm{~V}$ from the $\mathrm{I} .57 \mathrm{~V}$ band gap.

Considering the brief period of time that perovskite research has had to mature, it is likely that I.05 V is not the maximum value that a solar cell fabricated from $\mathrm{CH}_{3} \mathrm{NH}_{3} \mathrm{P}$ $\mathrm{bI}_{3}$ can attain for $V_{O C}$. A larger $V_{O C}$ is also predicted by the lower $15 \mathrm{meV}$ reported value for $E_{u}$. Some reasons for the discrepancy between reported data and measured data in- 
clude high noise levels-ideally we would measure the absorption tail through another order of magnitude to get a more accurate number-as well as difficulties forming a film thick enough to see a high signal in PDS measurements.

\section{CONCLUSIONS AND FUTURE WORK}

The values measured using photothermal deflection spectroscopy for the minimum Urbach energy of lead sulfide quantum dots and lead iodide perovskites are $98 \mathrm{meV}$ and $45 \mathrm{meV}$, measured over a maximum of two orders of magnitude. These values lead to a good agreement between the calculated open circuit voltage and the reported values attained by solar cells fabricated with these materials. Although a little bit more work needs to be done to obtain a more precise determination of the Urbach tail with these materials with absorption data extending over more orders of magnitude, the initial results are very promising in showing that the consideration of the Urbach tail is a very valuable input to choose the best materials for solar cells.

\section{ENDNOTES}

I. Nozik, 2002.

2. Brown et al., 2014 .

3. Green et al., 20I4.

4. Stranks et al., 20I3.

5. Shockley et al., I96I.

6. Guyot-Sionnest, 20I2

7. De Wolf et al., 20I4.

8. Hines, 2003.

9. Lu et al., 2009 .

IO. Im et al., 2012.

II. Yoon et al., 20I3.

I2. Erslev et al., 2012.

I3. Guyot-Sionnest et al., 2012.

I4. Ryu et al., 2014 .

I5. Lee et al., 20I2.

\section{REFERENCES}

Nozik, A.J. "Quantum Dot Solar Cells," Physica E. 2002. I4, II5-I2O.

P.R. Brown, D. Kim, R.R. Lunt, N. Zhao, M. Bawendi, J. C. Grossman, and V. Bulovi, "Energy Level Modification in Lead Sulfide Quantum Dot Thin Films through Ligand Exchange," ACS Nano 20I4. 8, 5863-5872.

Green, M.A., Ho-Baille, A., Snaith, H.J. Nat. Photon., 2014. $8,506-514$.
S. Stranks, G. Eperon, G. Grancini, C. Menelaou, M. Alococer, T. Leijtens, L. Herz, A. Petrozza, H. Snaith. "Electron-hole diffusion lengths exceeding I micrometer in an organometal trihalide perovskite absorber." Science, 2013 . $342,342-343$.

Shockley W. and Queisser, H.J. "Detailed Balance Limit of Efficiency of p-n Junction Solar Cells." J. Appl. Phys. I96I, 32, 510 .

Guyot-Sionnest, P. "Electrical Transport in Colloidal Quantum Dot Films.” J. Phys. Chem. Lett. 2012. 3, II69-II75.

De Wolf, S., Holovsky, J., Moon, S.J., Löper, P., Niesen, B., Ledinski, M., Haug, F.J., Yum, J.H., Ballif, C. "Organometallic Halide Perovskites: Sharp Optical Absorption Edge and Its Relation to Photovoltaic Performance." J. Phys. Chem. Lett. 2014. 5, I035- I039.

Hines, M.A.; Scholes G.D. "Colloidal PbS Nanocrystals with Size-Tunable Near-Infrared Emission: Observation of Post-Synthesis Self-Narrowing of the Particle Size Distribution." Adv. Mater. 2003. I5, I844-1849.

Lu, S.; Lingley, Z.; Asano, T.; Harris, D.; Barwicz, T.; Guha, S.; Madhukar, A. "Photocurrent Induced by Nonradiative Energy Transfer from Nanocrystal Quantum Dots to Adjacent Silicon Nanowire Conducting Channels: Toward a New Solar Cell Paradigm.” Nano. Lett. 2009. 9(I2), 45484552.

Im, J.-H.; Chung, J.; Kim, S.-J.; Park, N.-G. "Synthesis, Structure, and Photovoltaic Property of a Nanocrystalline $2 \mathrm{H}$ Perovskite-Type Novel Sensitizer $\left(\mathrm{CH}_{3} \mathrm{CH}_{2} \mathrm{NH}_{3}\right)$ PbI3." Nanoscale Res. Lett. 20I2. 7, 353, I-7.

Yoon, W.; Boercker, J. E.; Lumb, M. P.; Placencia, D.; Foos, E. E.; Tischler, J. G. "Enhanced Open-Circuit Voltage of PbS Nanocrystal Quantum Dot Solar Cells.” Sci. Rep. 2013. 3, 2225 .

P.T. Erslev, H.Y. Chen, J. Gao, M.C. Beard, A.J. Frank, J. van de Lagemaat, J.C. Johnson, J.M. Luther. “Sharp Exponential Tails in Highly Disordered Lead Sulfide Quantum Dot Arrays." Phys. Rev. B 2012. 86, I55313.

P. Guyot-Sionnest, E. Lhuillier, H. Liu. "A mirage study of CdSe colloidal quantum dot films, Urbach tail, and surface states." J. Phys. Chem. 2012. I37, I54704. 
S. Ryu, J.H. Noh, N.J. Jeon, Y.C. Kim, W.S. Yang, J. Seo, S.I. Seok. "Voltage output of efficient perovskite solar cells with high open-circuit voltage and fill factor." Energy Environ. Sci. 20I4. 7, $26 \mathrm{I} 4$.

M. Lee, J. Teuscher, T. Miyasaka, T.N. Murakami, H.J. Snaith. "Efficient Hybrid Solar Cells Based on Meso-Superstructured Organometal Halide Perovskites." Science, 2012. 338, 643-647.

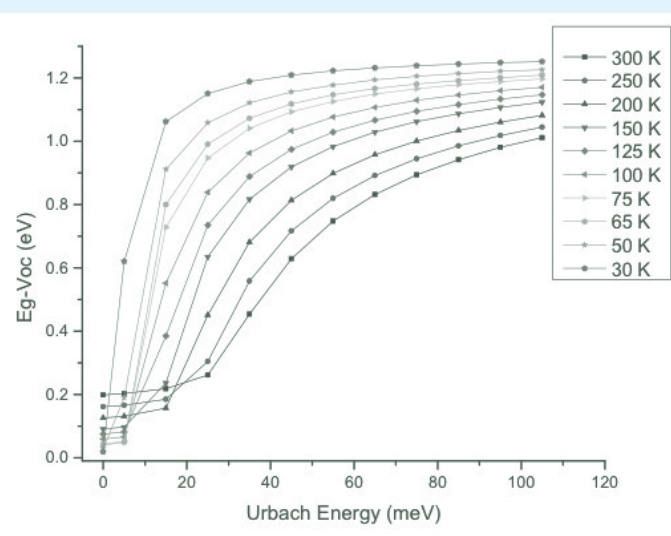

FIGURE 1: VOLTAGE DROP FROM BAND GAP ENERGY VS URBACH ENERGY

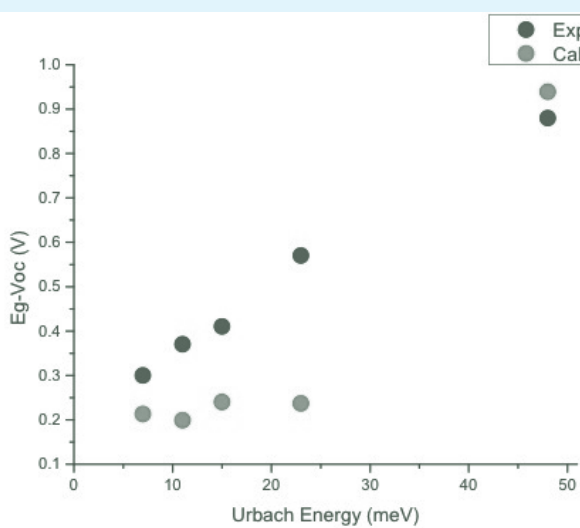

FIGURE 2: VOLTAGE DROP FROM BAND GAP ENERGY VS. URBACH ENERGY

\begin{tabular}{|l|l|l|l|l|}
\hline Material & Eg $(\mathrm{eV})$ & Eu $(\mathrm{meV})$ & Eg-VOC $(\mathrm{eV})$ (experiment) & Eg-VOC (eV) (calculated) \\
\hline $\mathrm{GaAs}$ & I.42 & 7 & 0.3 & $0.2 \mathrm{I} 3$ \\
\hline c-Si & I.I2 & II & 0.37 & 0.199 \\
\hline $\mathrm{CH}_{3} \mathrm{NH}_{3} \mathrm{PbI}_{3}-\mathrm{xClx}$ & I.57 & I5 & $0.4 \mathrm{I}$ & 0.24 \\
\hline $\mathrm{CIGS}$ & I.18 & 23 & 0.57 & 0.237 \\
\hline a-Si & I. 55 & 48 & 0.88 & 0.939 \\
\hline
\end{tabular}




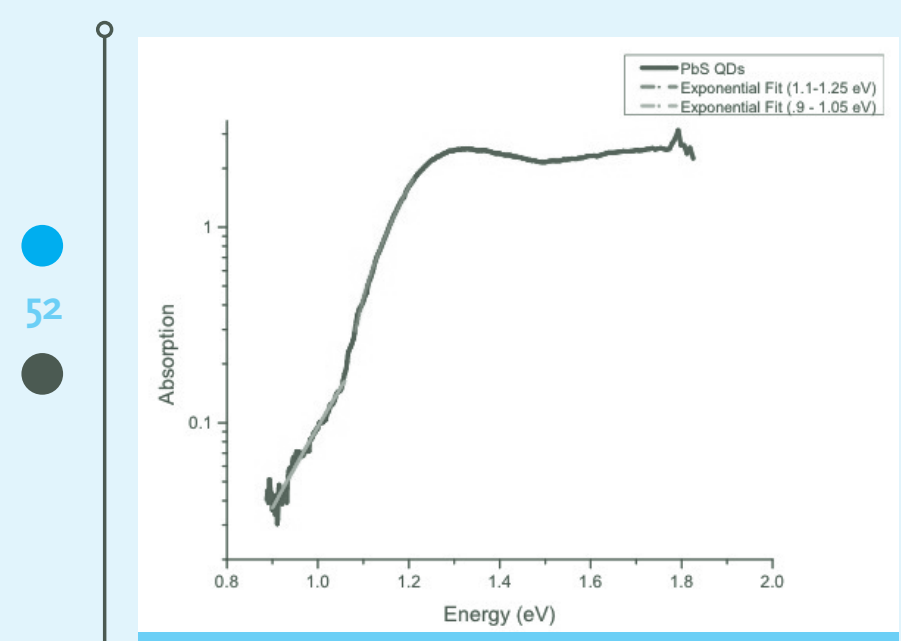

FIGURE 3: ABSORPTION VS. ENERGY

\begin{tabular}{|l|l|l|}
\hline Size $(\mathrm{nm})$ & Eg $(\mathrm{V})$ & VOC $(\mathrm{V})$ \\
\hline 2.9 & I.40 & 0.692 \\
\hline 3.1 & I.30 & 0.657 \\
\hline 3.3 & I.24 & 0.629 \\
\hline 3.5 & I.I8 & 0.602 \\
\hline 3.8 & I.09 & 0.545 \\
\hline 4.0 & I.05 & 0.542 \\
\hline 4.1 & I.03 & 0.483 \\
\hline
\end{tabular}

TABLE 2

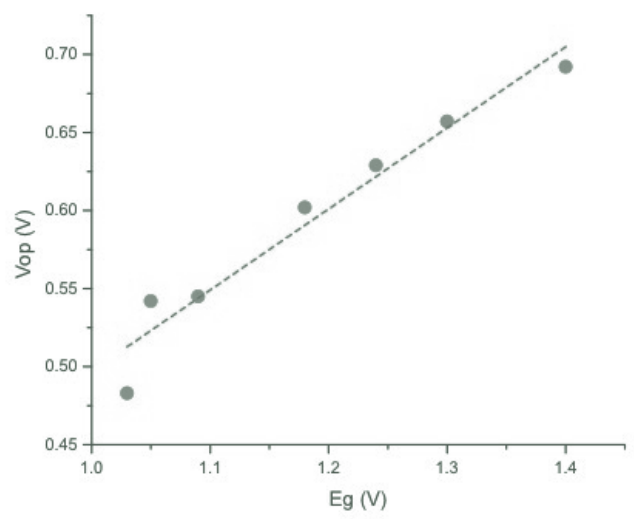

FIGURE 4: OPEN CIRCUIT VOLTAGE VS. ENERGY

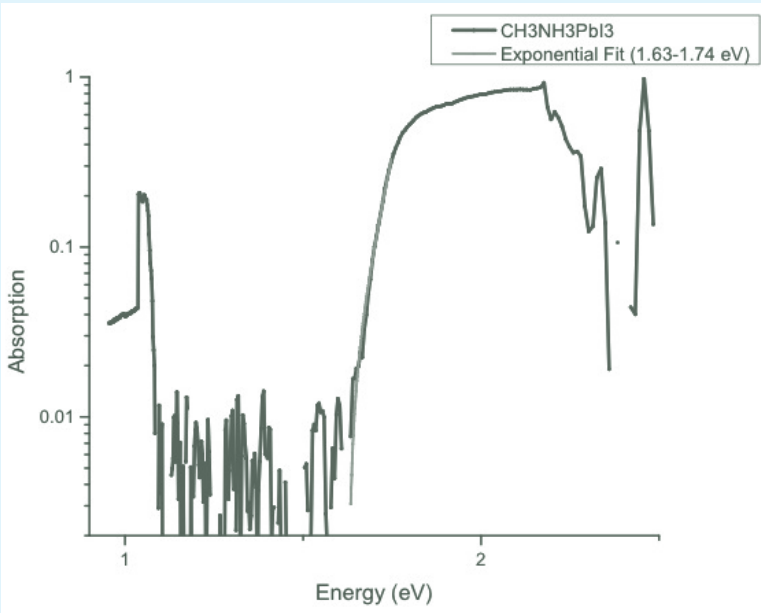

FIGURE 5: ABSOPRTION VS. ENERGY

ELEMENTS : : SPRING 2015 